\title{
Incorporación de nitrógeno para mejorar la eficiencia de uso del cultivo de arroz (Oryza sativa L.) en La Libertad, Perú
}

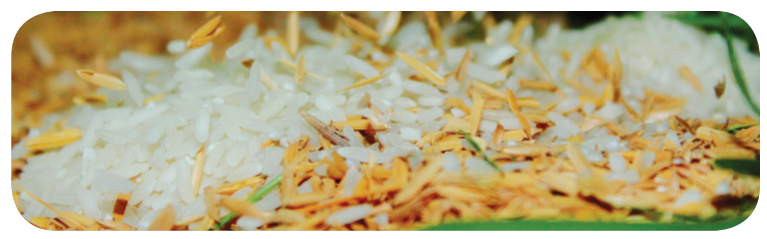

\author{
Elizabeth Consuelo Heros Aguilar ${ }^{1 *}$ \\ Hugo Soplín Villacorta ${ }^{2}$ \\ Guillermo Sosa Peralta ${ }^{3}$
}

Recepción: 23/05/2018 Aceptación: 17/07/2018

\section{Resumen}

El mejoramiento de la eficiencia de uso de nitrógeno reduciría el consumo de fertilizantes nitrogenados y contribuiría a la preservación del ambiente y al desarrollo de sistemas de producción sostenibles. Para mejorar la eficiencia de uso, se realizó un experimento en el valle del distrito de Jequetepeque, provincia de Pacasmayo, departamento de La Libertad en Perú, para identificar la forma de aplicación y los niveles de nitrógeno más adecuados para optimizar la eficiencia y disminuir las pérdidas que se originan por el mal manejo del nitrógeno.

Se evaluaron tres formas de aplicación del fertilizante nitrogenado (urea): Forma 1: 100\% incorporado en suelo seco; Forma 2: 50\% incorporado en suelo seco + 50\% al voleo al inicio del primordio floral en lámina de agua; y Forma 3: Voleo tradicional aplicando la mitad del nitrógeno a los quince días después del trasplante en lámina de agua y el 50\% restante al inicio del primordio floral al voleo en lámina de agua.

Los niveles utilizados fueron: 120,240 y $320 \mathrm{KgNha}^{-1}$ y un testigo 0 . El rendimiento en grano obtenido varió de 5.67 a 4.88tha-1, para formas y de 7.36 a 5.34tha ${ }^{-1}$, para niveles de nitrógeno. La estimación de la eficiencia de uso de nitrógeno a través de los indicadores de eficiencia agronómica, eficiencia fisiológica y factor parcial de productividad, identificaron que la forma 2 tuvo la mejor respuesta.

Palabras clave: Nitrógeno, niveles, formas de aplicación, eficiencia de uso de nitrógeno.

\begin{abstract}
The improvement of the efficient use of nitrogen would reduce the consumption of nitrogen fertilizers and contribute to the preservation of the environment and the development of sustainable production systems. To improve the efficiency of use, an experiment was conducted in the valley of Jequetepeque district, province of Pacasmayo, department of La Libertad in Peru, to identify the application form and the most adequate nitrogen levels to optimize efficiency and reduce losses that are caused by poor nitrogen management.
\end{abstract}

Three ways of nitrogenous fertilizer applications were evaluated (urea): Form 1: 100\% incorporated to dry soil; Form 2: 50\% incorporated to dry soil $+50 \%$ to the traditional at the beginning of the floral primordio in the water sheet; and Form 3: Traditional volley applying the half of nitrogen fifteen days later from the transplantation in the water sheet and the $50 \%$ left was applied at the beginning of the floral primordio volley in the water sheet.

The levels used were: 120,240 and $320 \mathrm{KgNha}-1$ and a control 0 . The obtained grain yield varied from 5.67 to 4.88tha-1, for forms and 7.36 to 5.34 tha-1, for nitrogen levels. The estimation of the efficiency of nitrogen use through the indicators of agronomic, and physiological efficiency and partial factor of productivity, identified that form 2 had the best response.

Key words: Nitrogen, levels, forms of application, efficiency of nitrogen use.

\footnotetext{
1. *Magister Scientiae, Profesora Asociada, Departamento de fitotecnia, Facultad de Agronomía, Universidad Nacional Agraria La Molina, LimaPerú; Autor para correspondencia: lizheros@lamolina.edu.pe

2. PhD. En Producción de semillas, Profesor Principal, Departamento de fitotecnia, Facultad de Agronomía, Universidad Nacional Agraria La Molina, Lima-Perú; email: husovi@lamolina.edu.pe

3. Magister Scientiae especialista en Estadística Aplicada, Coordinador, Dirección de Gestión de la Innovación Agraria, Instituto Nacional de Innovación Agraria, Perú; email: gsosa@inia.gob.pe
} 


\section{Introducción}

El arroz es uno de los cereales más importantes a nivel mundial por su gran contribución a la alimentación humana. Actualmente su producción enfrenta dos grandes desafios: 1) atenuar la reducción de los rendimientos por efecto del cambio climático, a fin de satisfacer las mayores demandas para suplir las nuevas necesidades de la población; y 2) Optimizar los niveles de nitrógeno aplicados al cultivo para reducir las emisiones de gases de efecto invernadero. La producción mundial es de aproximadamente 741 millones de toneladas, con un rendimiento promedio de 4.64tha ${ }^{-1}$, ocupando una superficie de 159 ' 807,722 has (FAO, 2017). Perú, durante el año 2014, cosechó una superficie de 381 368ha, en las que se produjo 2,896,613t con un rendimiento promedio de 7.6 tha $^{-1}$ (MINAGRI, 2017).

La producción de este alimento demanda altos niveles de nitrógeno (240 a 320KgNha-1) que se aplican en forma de urea o sulfato de amonio. El International Fertilizer Industry Association (IFA, 2014), señala que el uso de los fertilizantes a nivel mundial se ha incrementado en más de cinco veces en los últimos cincuenta años. De acuerdo a lo indicado por la IFA (2014), en el año 2012 se aplicaron en 1563 millones de hectáreas(Mha), 179 millones de toneladas métricas (Mt) de fertilizantes. El consumo global de fertilizantes fue de 109Mt de nitrógeno $(\mathrm{N})$, 41Mt de fosfato $\left(\mathrm{P}_{2} \mathrm{O}_{5}\right)$ y $29 \mathrm{Mt}$ de potasio $\left(\mathrm{K}^{2} \mathrm{O}\right)$.
Las estadísticas mundiales del año 2010 indican que la producción de arroz utiliza el 15\% del fertilizante nitrogenado a nivel global y emplea el $13 \%$ de fósforo y potasio (Heffer, 2013). Como insumo representan entre el 15 a 30\% del costo de producción en el sistema irrigado en el Asia (Moya et al., 2004; Pampolino et al., 2007).

Gran parte del nitrógeno aplicado en inundación se pierde como gas en la atmósfera. Por lo general 1/3 del nitrógeno aplicado en los suelos inundados del Asia, se pierde a las dos semanas a la atmósfera y $1 / 3$ del mismo permanece en suelo (Buresh, 2007). De acuerdo a lo señalado por Ladha et al., 2005 y Roberts 2008, la perdida de nitrógeno puede ser de 70 a $80 \%$ en condiciones de secano y de 60 a $70 \%$ en condiciones irrigadas cuando no existe un manejo apropiado del elemento.

La optimización del nitrógeno requiere de nuevos tipos de planta, adecuados a los diferentes ambientes y a distintos manejos del cultivo. Los cambios de tipo de planta, en los nuevos cultivares, están mejorando la eficiencia agronómica del nitrógeno, por la mayor formación de biomasa y mayores índices de cosecha (Schnnier et al., 1990). Está demostrado que, para reducir las pérdidas de este elemento, el fertilizante puede ser incorporado en el suelo, en vez de volearlo (Youndahl et al., 1986). En el Perú, el nitrógeno aplicado como urea o sulfato de amonio tiene una 
recuperación de 35-40\% (Ramírez y Sánchez, 1971), aplicado al voleo en lámina de agua. Incrementar este nivel de uso es uno de los desafíos más difíciles que deben abordar los investigadores (Thompson, 2012). Este proyecto determinó la eficiencia de uso del nitrógeno en tres formas de aplicación y cuatro niveles de nitrógeno con el cultivar IR-43.

\section{Materiales y Métodos}

El experimento se instaló en el fundo Luzben, con coordenadas $07^{\circ} 20^{\prime}$ 58" de latitud sur y $79^{\circ} 27^{\prime} 26^{\prime \prime}$ de longitud oeste, en la parte media del valle Jequetepeque, ubicado en la región La Libertad, provincia de Pacasmayo, en el distrito San José, a 106 m.s.n.m.
El suelo tuvo una textura franca (52\% de arena, $28 \%$ de limo y $20 \%$ de arcilla), con un $\mathrm{pH}$, de 6.55 (ligeramente ácido a neutro), y un contenido de materia orgánica de $1.06 \%$. El cultivar utilizado fue IR-43, desarrollado por el Instituto Internacional de Investigaciones en Arroz (IRR), planta semienana con 150 días de maduración. Se empleó el diseño de parcelas divididas con tres repeticiones, colocándose las formas de aplicación en parcelas y los niveles de nitrógeno en sub-parcelas, que tuvieron una dimensión de $15 \mathrm{~m} 2$ (3x5).

\section{Tratamientos}

Los tratamientos estudiados se detallan en la Tabla 1.

Tabla 1. Formas y niveles de aplicación de Nitrógeno en $\mathrm{Kgha}^{-1}$

\begin{tabular}{|c|l|c|c|c|c|}
\hline \multicolumn{2}{|c|}{ Formas } & \multicolumn{3}{c|}{ Niveles de nitrógeno } \\
\cline { 2 - 5 } F1 & N0=0 & N1=120 & N2=240 & N3=360 \\
\hline & $\begin{array}{l}\text { Cien por ciento incorporado a la preparación del } \\
\text { suelo en seco (I 100 PS) }\end{array}$ & 0 & 120 & 240 & 320 \\
\hline $\begin{array}{l}\text { Incorporado el 50\% a la preparación del sue- } \\
\text { fo en seco + voleo del 50\% restante al inicio } \\
\text { del primordio floral (IPF) en lámina de agua } \\
\text { (150 PS+V50IPF) }\end{array}$ & 0 & 60 & 120 & 160 \\
\hline \multirow{2}{*}{$\begin{array}{l}\text { Cincuenta por ciento al voleo a los quince días } \\
\text { después del transplante en lámina de agua+50\% } \\
\text { al voleo al inicio del primordio floral (V50 15 } \\
\text { DDT + V50 IPF)-(aplicación convencional) }\end{array}$} & 0 & 60 & 120 & 160 \\
\hline
\end{tabular}




\section{Manejo agronómico}

El sistema de siembra fue al trasplante, $y$ se realizó con plántulas de treinta días de edad, con golpes de cuatro a cinco plántulas a $0.25 \times 0.25 \mathrm{~m}$, en hileras de $5 \mathrm{~m}$ de largo. La densidad fue de 16 golpes $\mathrm{m} 2$. Los riegos fueron de inundación continua y el manejo del agua fue individual por sub parcela.

La incorporación de nitrógeno se realizó en suelo seco. La fuente nitrogenada fue urea. El fósforo $\left(60 \mathrm{Kgha}^{-1}\right)$ se aplicó como superfosfato triple de calcio y el potasio como sulfato de potasio $\left(60 \mathrm{Kgha}^{-1}\right)$, que fueron aplicados en su totalidad luego de la incorporación del nitrógeno.
Finalizada la aplicación de los fertilizantes, cada sub-parcela fue regada, luego trasplantada, sin batir el suelo. Para el control de malezas se aplicó butachlor a los cinco días después del trasplante. La cosecha por subparcela fue de $8 \mathrm{~m}^{2}$, que se realizó en forma manual. Los rendimientos se ajustaron a $14 \%$ de humedad.

\section{Cálculos dela eficiencia de uso de nitrógeno-EUN}

La eficiencia de uso de nitrógeno es la medida de la ganancia en producción de grano, por unidad de nutriente aplicado (Dobermann, 2007).

a. Eficiencia agronómica - EA. Son los kilogramos de aumento en la producción por kilogramos de nitrógeno aplicado. La fórmula usada fue:

$$
\text { Eficiencia agronómica: } \frac{\text { Rdto (nivel de N) - Rdto (nivel } 0 \text { de N) }}{\text { Nivel de Nitrógeno aplicado }}
$$

b. Eficiencia fisiológica - EF. Son los kilogramos de aumento de la producción por kilogramos de aumento en la absorción de nitrógeno. La fórmula usada fue:

$$
\text { Eficiencia fisiológica: } \frac{\text { Rdto (nivel de N) - Rdto (nivel } 0 \text { de N) }}{\mathrm{N} \text { absorbido (nivel de } \mathrm{N})-\mathrm{N} \text { absorbido (nivel de } 0 \text { de } \mathrm{N} \text { ) }}
$$

c. Factor parcial de productividad - FPP. Se define como el rendimiento del cultivo por unidad de fertilizante aplicado. La fórmula usada fue:

$$
\text { Factor parcial de productividad: } \quad \frac{\text { Rdto (nivel de } \mathrm{N} \text { ) }}{\text { Nivel de } \mathrm{N} \text { aplicado }}
$$




\section{Determinación de la acumulación de nitrógeno}

Se seleccionaron muestras de diez tallos, incluyendo hojas y granos en dos repeticiones. Esta determinación se realizó en el Laboratorio de Suelos de la Universidad Nacional Agraria La Molina, en Perú. Se usó el método de Kjeldahl para determinar el porcentaje de nitrógeno en la paja y en los granos. El porcentaje de nitrógeno en la paja y el grano se multiplicó por la respectiva materia seca de cada uno de estos, obtenida a la cosecha. Finalmente se sumaron los valores obtenidos para calcular el nitrógeno total absorbido por la planta en $\mathrm{Kgha}^{-1}$.

\section{Resultados y Discusión}

\section{Rendimiento en grano}

El análisis de variancia (Tabla 2) indica que existe significación estadística para formas de aplicación y niveles de nitrógeno, mientras que para la interacción formas y niveles no existe significación. Los coeficientes de variabilidad del experimento fueron $7.43 \%$ (error a) y $7.47 \%$ (error b), que indican confiabilidad de los resultados (Ver tabla 2).

En formas de aplicación de nitrógeno, el mayor rendimiento se alcanzó con la forma 2 (I50 PS+V50IPF), con 5,67tha ${ }^{-1}$, siendo superior estadísticamente a la forma 3 (V50 15 DDT + V50 IPF), que produjo un rendimiento promedio de 4.88tha ${ }^{-1}$, y a la forma 1 (I 100 PS), que tuvo un rendimiento de 5.14tha ${ }^{-1}$ (Tabla 3). Estos resultados concuerdan con lo señalado por Jaramillo et al. (2008), quienes exponen que cuando la urea se aplica en suelo seco, la superficie de contacto de las arcillas (complejo de cambio o adsorción) están totalmente receptivas a la atracción de cationes $\mathrm{NH}_{4}$ (Ver tabla 3).

Tabla 2. Análisis de variancia del rendimiento en grano

\begin{tabular}{|c|c|c|c|c|c|c|}
\hline FV & GL & S.C & C.M & F cal & Pr $>$ F & Nivel de significación \\
\hline Bloques & 2 & 3.03 & 1.515 & 15,500 & 0.0001 & $\star *$ \\
\hline Formas & 2 & 2.51 & 1.254 & 12,830 & 0.0003 & $\star *$ \\
\hline Error (a) & 4 & 0.39 & 0.097 & 1,000 & & \\
\hline Niveles & 3 & 121.34 & 40.447 & 413,910 & $<.0001$ & $* \star$ \\
\hline F $^{\star} \mathbf{N}$ & 6 & 0.74 & 0.123 & 1,260 & 0.324 & NS \\
\hline Error (b) & 18 & 1.76 & 0.098 & & & \\
\hline Total & 35 & 129.77 & & & & \\
\hline
\end{tabular}

$\mathrm{CV}($ error $\mathrm{a})=7.43 \% ; \mathrm{CV}($ error $\mathrm{b})=7.47 \%$ 
Tabla 3. Rendimiento en grano para formas de aplicación y niveles de nitrógeno

\begin{tabular}{|c|c|c|}
\hline Tratamientos & & Rendimiento Kgha $^{-1}$ \\
\hline \multirow{3}{*}{ Formas de Aplicación } & $\mathrm{F} 2$ & $5,672 \mathrm{a}^{*}$ \\
\cline { 2 - 3 } & $\mathrm{F} 1$ & $5,144 \mathrm{~b}$ \\
\cline { 2 - 3 } & $\mathrm{F} 3$ & $4,878 \mathrm{~b}$ \\
\hline \multirow{4}{*}{ Niveles de N } & 320 & $7,358 \mathrm{a}$ \\
\cline { 2 - 3 } & 240 & $6,769 \mathrm{~b}$ \\
\cline { 2 - 3 } & 120 & $5,343 \mathrm{c}$ \\
\cline { 2 - 3 } & 0 & $1,464 \mathrm{~d}$ \\
\hline
\end{tabular}

*Para columnas dentro de formas de aplicación y niveles de nitrógeno, los datos que tienen una letra en común no son significativamente diferentes entre sí, según la prueba Duncan, al nivel de 5\% de probabilidad.

Las respuestas en rendimiento a nivel 0 , es decir sin aplicación de fertilizante nitrogenado, fue en promedio de $1.46 \mathrm{t} \mathrm{ha-1}$, siendo el nivel de $320 \mathrm{KgNha}^{-1}$, el que presentó el mayor rendimiento (7.36tha-1); seguido de los niveles $240 \mathrm{y}$ 120 con 6.77 y 5.34 tha $^{-1}$, respectivamente.

Las respuestas de los rendimientos a los niveles de nitrógeno fueron de tipo cuadrático (figura 1). Los incrementos de rendimiento entre los niveles 120, 240 y 320 fueron de 365, 462 y $503 \%$ en relación al nivel 0 , respectivamente. Sin embargo, este incremento del rendimiento debido al aumento del nivel de nitrógeno no es ilimitado, sino que alcanza un valor máximo y sigue una tendencia decreciente (Baker et al., 2004). (Ver figura 1).

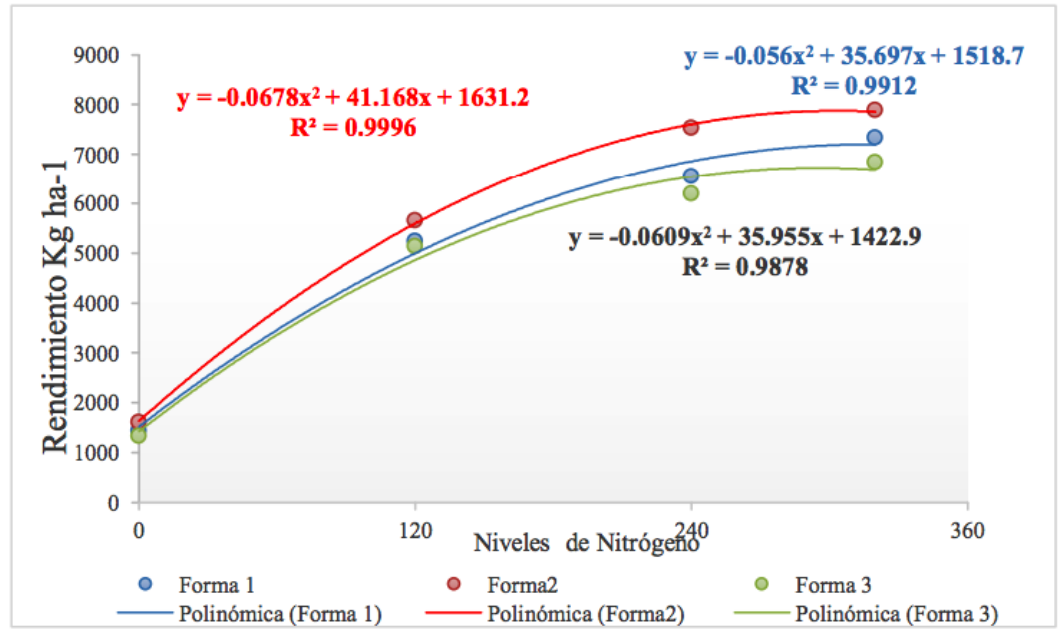

Figura 1. Relación entre niveles de nitrógeno y rendimiento en grano. 
Los rendimientos relativamente bajos presentados en este estudio, probablemente se debieron a temperaturas altas, estrés hídrico y resurgencia de sogata (Tagosodes oryzicolus), vector del virus de hoja blanca, que afectaron la campaña agrícola en que se realizó esta investigación.

\section{Determinación de la biomasa}

Las determinaciones de la biomasa se realizaron en los estadios de máximo macollamiento, inicio de primordio floral (IPF), floración y madurez fisiológica del grano, por ser los estadios de mayor contribución al rendimiento. La figura 2 indica que la producción de materia seca se incrementa hasta la madurez fisiológica de los granos. Entre formas, la incorporación total (F1) alcanzó mayor biomasa que las demás formas (F2 y F3). Chaturvedi (2005), señala que la acumulación de materia seca se incrementa significativamente con la aplicación de fertilizante nitrogenado en todos los estadios de crecimiento del cultivo. (Ver figura 2).

La prueba Duncan realizada a la variable producción de materia seca en el estadio de madurez fisiológica indica que, entre las formas de aplicación, no hay significación estadística. Sin embargo, para el caso de los niveles, existen diferencias significativas entre las cuatro dosis, ocupando el primer lugar la dosis de $320 \mathrm{KgN}$ con $22,327 \mathrm{Kgha}^{-1}$. Las dosis de 240, 120 y 0 produjeron 19657,12735 y 8 $202 \mathrm{Kg} \mathrm{ha}^{-1}$ de materia seca, respectivamente (Tabla 4).

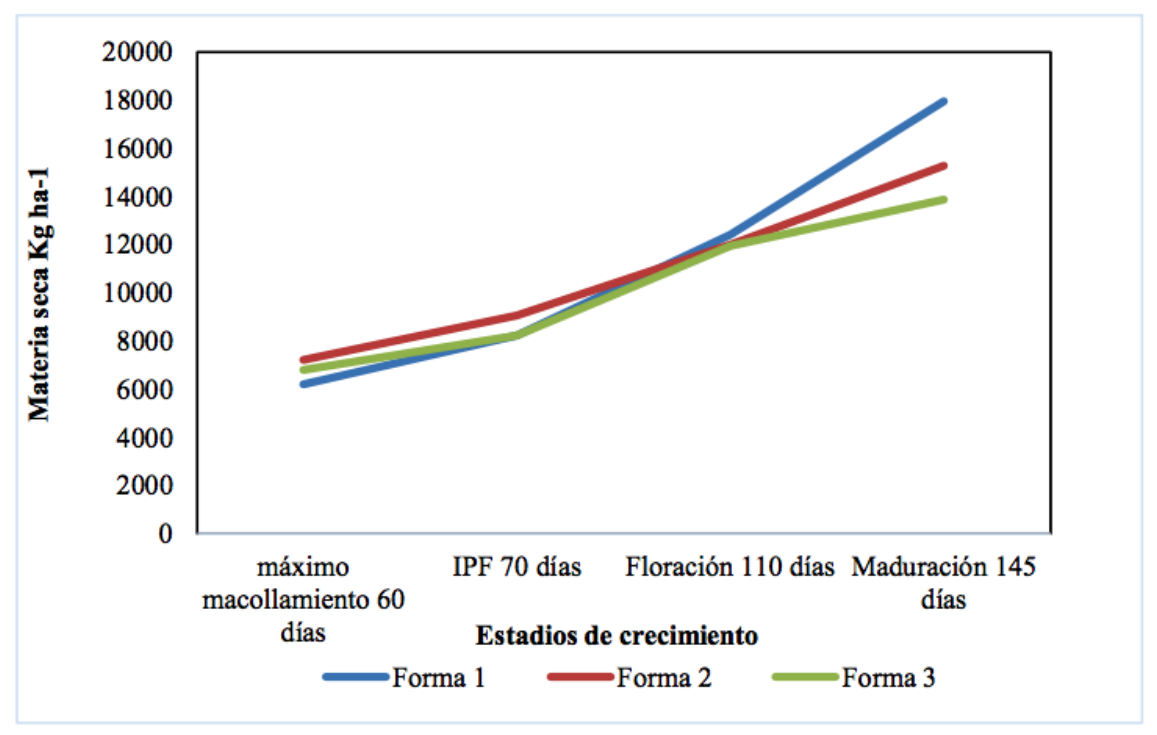

Figura 2. Producción de materia seca en cuatro estadios de desarrollo para formas de aplicación de nitrógeno. 
Tabla 4. Producción de materia seca en cuatro estadíos de desarrollo para formas de aplicación de nitrógeno

\begin{tabular}{|c|c|c|}
\hline Tratamientos & & Materia seca Kgha-1 \\
\hline \multirow{3}{*}{$\begin{array}{c}\text { Formas } \\
\text { de aplicación }\end{array}$} & $\mathrm{F} 1$ & $17,954 \mathrm{a}^{*}$ \\
\cline { 2 - 3 } & $\mathrm{F} 2$ & $15,268 \mathrm{a}$ \\
\cline { 2 - 3 } & $\mathrm{F} 3$ & $13,865 \mathrm{a}$ \\
\hline Niveles de N & 320 & $22,327 \mathrm{a}$ \\
\cline { 2 - 3 } & 240 & $19,657 \mathrm{~b}$ \\
\cline { 2 - 3 } & 120 & $12,735 \mathrm{c}$ \\
\cline { 2 - 3 } & 0 & $8,202 \mathrm{~d}$ \\
\hline
\end{tabular}

*Para columnas dentro de formas de aplicación y niveles de nitrógeno, los datos que tienen una letra en común no son significativamente diferentes entre sí, según la prueba Duncan, al nivel de 5\% de probabilidad.

\section{Relación entre materia seca y rendimiento}

Hubo una alta relación entre biomasa y el rendimiento en grano. Los valores de los coeficientes de correlación fueron: 0.98, 0.99 y 0.97 para F2, F1 y F3 respectivamente a la madurez fisiológica de los granos; y tuvo el mayor peso en la F1 con 17.95tha ${ }^{-1}$. Para F2 y F3 los pesos fueron de 15.27 y 13.87 toneladas de materia seca por hectárea, correspondientemente.

Estudios realizados por Fageria (2007), señalan que la producción de materia seca tiene una alta y mayor correlación con el rendimiento en grano, durante los estadios de crecimiento de la panícula, floración y madurez fisiológica en comparación con estadios de crecimiento temprano.

\section{Altura de planta}

No se encontraron diferencias significativas para formas de aplicación. Las alturas fueron 95, 94 y $93 \mathrm{~cm}$ para F3, F2 y F1, respectivamente. Para el caso de los niveles de nitrógeno, las dosis de 320, 240 y $120 \mathrm{KgNha}^{-1}$, no mostraron diferencias entre ellos y tuvieron alturas de 95, 97 y $98 \mathrm{~cm}$ correspondientemente. El nivel 0 tuvo una altura de $86.5 \mathrm{~cm}$ y fue significativamente diferente a las demás dosis. Estos resultados son similares a los reportados por Somasudaran et al. (2002) y Chatuverdi (2005), que señalan que las plantas con las dosis de 150 y de $100 \mathrm{Kg}$ de nitrógeno por ha-1, alcanzan alturas mayores que con el nivel 0.

\section{Componentes de rendimiento}

La prueba de comparación de medias (Tabla 5) indica que, para formas de aplicación, el número de panículas por metro cuadrado $\left(\mathrm{m}^{2}\right)$, F2 fue superior a F1 y F3. Estos tratamientos no mostraron diferencias entre sí. En relación a número de granos por panícula, el porcentaje de granos llenos y peso de 1,000 granos, las tres formas de aplicación, no presentaron diferencias significativas.

Para niveles de nitrógeno, $320 \mathrm{KgNha}^{-1}$ fue superior a 240, 120 y 0 en número de panículas por metro cuadrado y número de granos panícula $^{-1}$. Para 240, 120 y 0 hubo también diferencias para estos componentes. Ottis et 
al. (2005), reportaron que existe una alta correlación entre rendimiento y número de panículas. En el porcentaje de granos llenos, no hubo diferencias entre 320 y 240, siendo ambos superiores a 120 y 0 . Para peso de 1,000 granos no existieron diferencias significativas (Ver tabla 5).

El análisis de regresión múltiple indica que la mejor igualdad para predecir el rendimiento en función a sus componentes es:

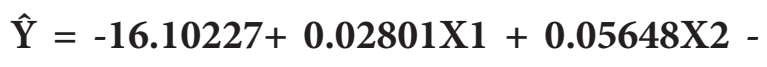

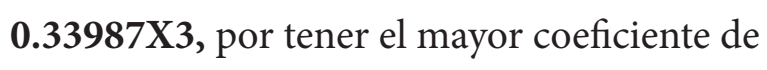
determinación múltiple $\left(\mathrm{R}^{2}=0,997\right)$, siendo $\mathrm{X} 1$ : número de panículas ha ${ }^{-1} ; \mathrm{X} 2$ : número de granos panículas ${ }^{-1} \mathrm{y} \mathrm{X} 3$ : peso de 1,000 granos.

\section{Índice de cosecha}

Este es la relación entre el rendimiento en grano y el rendimiento biológico. Esta variable tuvo una tendencia creciente conforme incrementaban las dosis de nitrógeno. Sin embargo, entre formas de aplicación no se encontraron diferencias significativas, pero sí en los niveles de nitrógeno. Las dosis de 320 (48\%) y 240 (45\%) fueron estadísticamente similares y superaron a las dosis de $120 \mathrm{y} 0 \mathrm{Kg}$ $\mathrm{Nha}^{-1}$., que tuvieron índices de cosecha de 42 y $25 \%$, respectivamente (figura 4).

Estudios realizados en Venezuela (Alfonzo et al., 2011) señalan que un alto índice de cosecha en los cultivares de arroz indican una me-

Tabla 5. Efecto de las formas de aplicación y niveles de $\mathrm{N}$ en los componentes del rendimiento en grano

\begin{tabular}{|c|c|c|c|c|c|}
\hline \multirow{2}{*}{ Tratamientos } & & $\begin{array}{c}\text { Número de } \\
\text { panículas por } \mathbf{~ m}^{-2}\end{array}$ & $\begin{array}{c}\text { Número } \\
\text { de granos } \\
\text { panicula }^{-1}\end{array}$ & $\begin{array}{c}\text { Porcentaje } \\
\text { de granos } \\
\text { llenos }\end{array}$ & $\begin{array}{c}\text { Peso de } \\
\mathbf{1 , 0 0 0} \text { granos }\end{array}$ \\
\hline \multirow{3}{*}{ Formas } & F2 & $273 \mathrm{a}^{*}$ & $140 \mathrm{a}$ & $84 \% \mathrm{a}$ & $26.72 \mathrm{a}$ \\
\cline { 2 - 6 } & $\mathrm{F} 1$ & $258 \mathrm{~b}$ & $139 \mathrm{a}$ & $83 \% \mathrm{a}$ & $26.59 \mathrm{a}$ \\
\cline { 2 - 6 } & $\mathrm{F} 3$ & $251 \mathrm{~b}$ & $137 \mathrm{a}$ & $85 \% \mathrm{a}$ & $25.89 \mathrm{a}$ \\
\hline \multirow{3}{*}{ Niveles de N } & 320 & $298 \mathrm{a}$ & $148 \mathrm{a}$ & $85 \% \mathrm{a}$ & $26.57 \mathrm{a}$ \\
\cline { 2 - 6 } & 240 & $287 \mathrm{~b}$ & $141 \mathrm{~b}$ & $84 \% \mathrm{a}$ & $26.48 \mathrm{a}$ \\
\cline { 2 - 6 } & 120 & $267 \mathrm{c}$ & $134 \mathrm{c}$ & $83 \% \mathrm{~b}$ & $26.37 \mathrm{a}$ \\
\cline { 2 - 6 } & 0 & $191 \mathrm{~d}$ & $139 \mathrm{~d}$ & $82 \% \mathrm{~b}$ & $26.19 \mathrm{a}$ \\
\hline
\end{tabular}

*Para columnas dentro de formas de aplicación y niveles de nitrógeno, los datos que tienen una letra en común no son significativamente diferentes entre sí, según la prueba Duncan a nivel de 5\% de probabilidad. 
nor pérdida de nitrógeno en la paja; y esto contribuye a una mayor eficiencia de uso de este componente. Los cultivares modernos de arroz tienen una mejor respuesta al uso de altas dosis de nitrógeno, y responden produciendo más grano que paja (Yoshida, 1981); pero hasta cierto nivel, más allá del cual los rendimientos bajan por mayor producción de paja y porque la planta se hace más sensible al ataque de insectos y enfermedades (Ver figura 3).

\section{Determinación de la eficiencia de uso}

\section{de nitrógeno}

La eficiencia de uso de nitrógeno es un concepto importante para evaluar los sistemas de producción. Puede ser altamente influenciada por el manejo de fertilizantes, suelo y la relación agua-planta y condiciones climáticas. Estudios en los últimos años señalan que aplicaciones excesivas de este elemento han ocasionado en la atmósfera deposiciones de NH3+ y NH4+ (Pearson and Stewart, 1993); y una acumulación excesiva en el suelo de $\mathrm{NH} 4+$ en concentraciones que varían de 2 a $20 \mathrm{~mm}$ y, en algunos casos, hasta valores muy altos como 40mm (Glass et al.,2002; Kronzucker et al., 2000).

Global Rice Science Partnership (2013), señala que las pérdidas de nitrógeno a partir de la volatilización del amonio pueden llegar a $50 \%$ o más en las regiones tropicales; mientras que, en las regiones templadas por la incorporación del nitrógeno al suelo, las pérdidas pueden ser insignificantes. Snyder (2009), señala que la urea aplicada al voleo y luego incorporada al suelo registró una pérdida de $\mathrm{NH}_{3}$ del 16\%, y del 27\% cuando se colocó en banda superficial (Ver tabla 6).

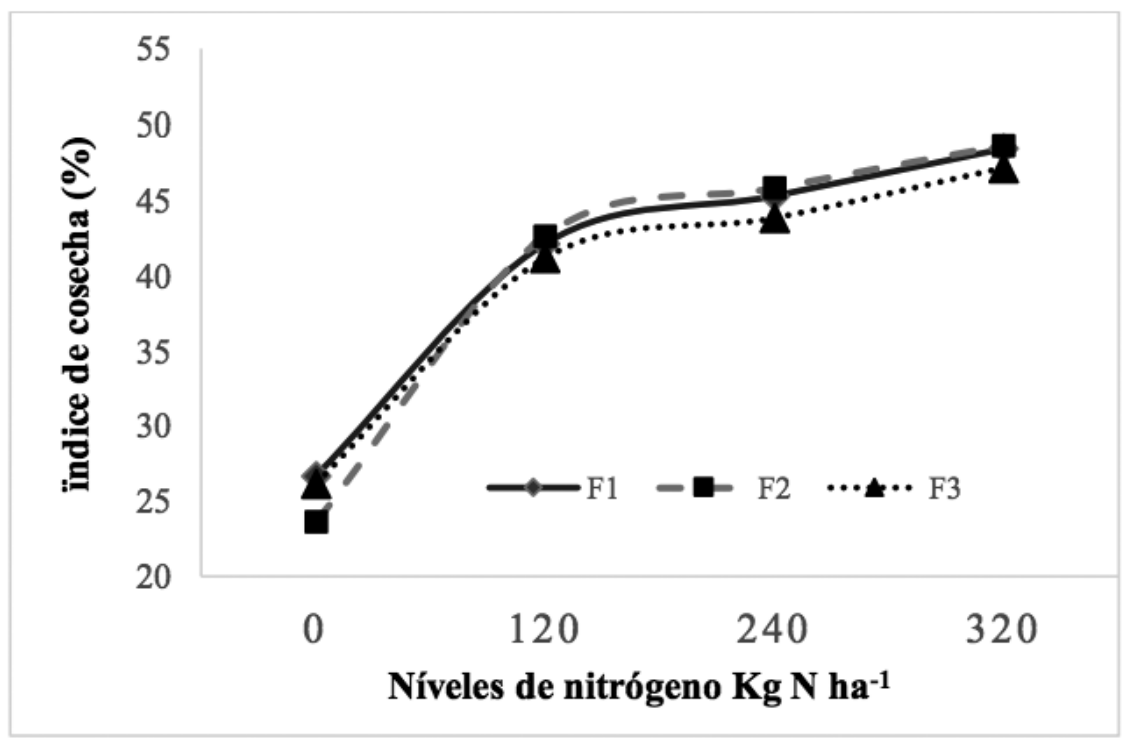

Figura 3. Índice de cosecha para tres formas de aplicación y cuatro niveles de nitrógeno 
Tabla 6. Eficiencia agronómica, eficiencia fisiológica y factor parcial de productividad del fertilizante para formas de aplicación y niveles de nitrógeno

\begin{tabular}{|c|c|c|c|}
\hline Tratamientos & $\begin{array}{l}\text { Eficiencia agronómica } \\
\text { Rdto. (nivel dado de ni- } \\
\text { trógeno) - Rdto (nivel 0)/ } \\
\text { Nivel de nitrógeno usado }\end{array}$ & $\begin{array}{l}\text { Eficiencia fisiológica } \\
\text { Rdto. (nivel dado de } \\
\text { nitrógeno)- Rdto } \\
\text { (nivel 0)/ nitrógeno } \\
\text { absorbido (en un ni- } \\
\text { vel dado - nitrógeno } \\
\text { absorbido (en nivel 0) }\end{array}$ & $\begin{array}{l}\text { Productividad Par- } \\
\text { cial del fertilizante } \\
\text { Rdto. (nivel dado de } \\
\text { nitrógeno)/ Nivel de } \\
\text { nitrógeno aplicado }\end{array}$ \\
\hline \multicolumn{4}{|l|}{ Formas } \\
\hline F1 & $24.80 \pm 1.89 \mathrm{~b}^{*}$ & $38.12 \pm 5.26 \mathrm{c}$ & $32.35 \pm 1.88 \mathrm{ab}$ \\
\hline $\mathrm{F} 2$ & $26.82 \pm 1.89 a$ & $54.26 \pm 5.26 \mathrm{a}$ & $35.02 \pm 1.88 \mathrm{a}$ \\
\hline F3 & $23.19 \pm 1.89 c$ & $42.54 \pm 5.26 \mathrm{~b}$ & $30.09 \pm 1.88 \mathrm{~b}$ \\
\hline C.V (\%) & 7.59 & 11.70 & 5.78 \\
\hline \multicolumn{4}{|l|}{$\begin{array}{c}\text { Niveles } \\
\text { de nitrógeno }\end{array}$} \\
\hline 120 & $33.35 \pm 1.7 \mathrm{a}$ & $71.89 \pm 6.64 \mathrm{a}$ & $45.44 \pm 1.84 \mathrm{a}$ \\
\hline 240 & $22.75 \pm 1.7 \mathrm{~b}$ & $36.41 \pm 6.64 \mathrm{~b}$ & $28.79 \pm 1.84 b$ \\
\hline 320 & $18.70 \pm 1.7 \mathrm{c}$ & $26.62 \pm 6.64 c$ & $23.23 \pm 1.84 c$ \\
\hline C.V (\%) & 6.81 & 14.71 & 5.67 \\
\hline
\end{tabular}

*Para columnas dentro de formas de aplicación y niveles de nitrógeno, los datos que tienen una letra en común no son significativamente diferentes entre sí, según la prueba Duncan a nivel de $5 \%$ de probabilidad.

Los resultados consignados en la Tabla 6 indican que F2 permite obtener la mayor eficiencia agronómica que las demás formas; le sigue F1 y finalmente F3. Los datos se presentan en un rango de 23.19 a 26.82. Estas eficiencias agronómicas se encuentran entre las obtenidas por Quintero et al. (2014), que reportan eficiencias de 18 a $26 \mathrm{Kg}$ grano por kilogramo de nitrógeno aplicado.
También se puede observar que conforme se incrementan los niveles de nitrógeno la eficiencia agronómica disminuye. Esto debido a una mayor pérdida de este elemento al ambiente que causa polución de la atmósfera y a los sistemas de agua como lo señalan Kondo et al. (2003). Belder et al. (2005), reportan rangos de eficiencia agronómica de 0 a $26 \mathrm{Kg}$ 
de grano por unidad de nitrógeno aplicado, señalando que existen otros factores que limitan la expresión de la eficiencia. Estudios previos realizados por Snyder (2009), reportan que la eficiencia agronómica del nitrógeno aplicado es de 10 a 30 unidades de grano de cereal por unidad de nitrógeno aplicado. Li et al. (2015), usando urea revestida, obtuvo una eficiencia agronómica de $17.7 \mathrm{Kg}$ grano $\mathrm{Kg}^{-1} \mathrm{~N}$.

La eficiencia fisiológica, en este estudio, fue mayor en F2 en comparación a las demás formas de aplicación. Para niveles de nitrógeno, la menor dosis $\left(120 \mathrm{KgNha}^{-1}\right)$ tuvo el valor más alto de eficiencia fisiológica. Estos resultados coinciden con lo señalado por Singh et al. (2014), quienes indican que el uso excesivo de nitrógeno resulta en detrimento de la eficiencia fisiológica. La eficiencia de uso de nitrógeno está limitada por la habilidad de las plantas para obtener el nitrógeno del fertilizante aplicado (Shrawat et al., 2008).

El factor parcial de productividad (FPP), en este estudio indicó que F2 es superior a F3, pero estadísticamente similar a F1. El rango de valores fue de 30.09 a 35.02 , valores que se encuentran por debajo de los niveles típicos en cereales (maíz, arroz y trigo), reportados por Fixen et al. (2015) (40-90 Kg de grano $\mathrm{Kg}^{-1}$ de nitrógeno aplicado, considerando que el suelo posee niveles de fósforo y potasio dentro del rango reco- mendado). Además, este bajo FPP, sugeriría que existe una respuesta baja del suelo a la sobre aplicación de nutrientes y a los efectos de estrés bióticos, registrados durante las etapas de crecimiento.

\section{Conclusiones}

La incorporación de nitrógeno en suelo seco tiene mayor respuesta en el rendimiento en grano que al voleo en capa de agua en el cultivo de arroz. La eficiencia de uso de nitrógeno evaluada a través de los indicadores de eficiencia agronómica, eficiencia fisiológica, factor parcial de productividad fueron más altos en los tratamientos de incorporación que el tratamiento de voleo en agua. La dosis de $240 \mathrm{KgNha}-1$ es la más adecuada por tener mejor eficiencia. Se hace un especial reconocimiento al Programa Nacional de Innovación Agraria (PNIA), por haber financiado esta investigación. 


\section{Referencias}

Alfonzo N.; España M.; López M.; Cabrera-Bisbal E. y Abreu P. (2011). Eficiencia de uso de nitrógeno en arroz de secano en un suelo ácido del occidente del Estado Guarico. Agronomía Trop. 61 (3-4): 215-220

Baker, D.A.; Young, D.L.; Huggins, D.R. y Pan, W.L. (2004). Economically optimal nitrogen fertilization for yield and protein in Hard Red Spring Wheat. Agron. J., 96: 126-133

Belder, P.; Spiertz, J.; Bouman, B.; Lu, G. y Tuong, P. (2005). Nitrogen economy and water productivity of lowland rice under water-saving irrigation. Field Crops Research 93(2-3): 169 - 185. DOI http://dx.doi.org/10.1016/j.fcr.2004.09.022

Buresh R.J. (2007). Fertile Progress. In: Rice Today. July-September

Chaturvedi, I. (2005). Effect of nitrogen fertilizers on growth, yield and quality of hybrid rice. J. Plant Nutr. 6:611-618

Dobermann, A. (2007). Nutrient use efficiency - measurement and management. In: IFAInternational Workshop on Fertilizer Best Management Practices. Brussels, Belgium, pp. 1-28

Fageria, N. (2007). Yield physiology of rice. Journal of Plant Nutrition, 30: 843-879

Fixen, P.; Brentrup, F.; Bruulsema T., W.; García, F.; Norton, R. y Zingore, Sh. (2015). Nutrient/ fertilizer use efficiency: Measurement, current situation and trend. Chap. 2. In: Drechsel, P., Heffer, P., Magen, H., Mikkelsen, R., Wichelns, D. (Eds.). Managing Water and Fertilizer for Sustainable Agricultural Intensifcation... p. 8-38.

Glass, ADM.; Britto, DT.; Kaiser, BN.; Kinghorn, JR.; Kronzucker, H.J.; Kumar, A.; Okamoto, M.; Rawat, S.; Siddiqi M.Y.; Unkles, S.E. y Vidmar, J.J. (2002). The regulation of nitrate and ammonium transport systems in plants. J Exp Bot 53:855-864. doi:10.1093/jexbot/53.370.855

Global Rice Science Partnership (2013). Rice almanac, 4th Edition. Los Baños (Philippines): International Rice Research Institute, p. 283 
Heffer, P. (2013). Assessment of fertilizer use by crop at the global level 2010-2010/11 International Fertilizer Industry Association, Paris, France. http://www.fertilizer.org/En/Statistics/Agriculture_Committee_Databases.aspx

Internationale Funkausstellung Berlin (2014). IFADATA. http://ifadata.fertilizer.org/ucSearch.aspx

Jaramillo, S.; Pulver, P. y Duque M.C. (2008). Efecto del manejo de la fertilización nitrogenada en arroz de riego, sobre la expresión del potencial de rendimiento de líneas élites y cultivares comerciales- unicauca.edu.co. Disponible en: https://scholar.google. com/scholar?oe=utf8\&client=firefoxb\&gfe_rd=cr\&um=1\&ie=UTF-8\&lr\&q=related:x_ osNaurczoyXM:scholar.google.com/

Kondo, M.; Pablico, P.P.; Aragones, D.V.; Agbisit, R.; Abe, J.; Morita, S. y Courtois, B. (2003). Genotypic and environmental variations in root morphology in rice genotype under upland field conditions. Plant Soil 255:189-200. doi:10.1023/ A:1026142904714

Kronzucker, H.J.; Siddiqi, M.Y.; Glass, A.D.M. y Kirk, G.J.D. (2000). Comparative kinetic analysis of ammonium and nitrate acquisition by tropical lowland rice: implications for rice cultivation and yield potential. New Phytol 145:471-476. doi:10.1046/j.1469-8137.2000. 00606.x

Ladha, J.K.; Pathak, H.; Krupnik, T.J.; Six, J. y van Kessel, C. (2005). Adv. Agron. 87: 85-156

Li, D.; Xu, M.; Qin, H.; Shen, H.; Sun, N.; Hosen, Y. y He, X. (2015). Polyolefin-coated urea improves nitrogen use efficiency and net profitability of rice-rice cropping systems. International Journal of Agriculture \&Biología. DOI. 10.17957/IJAB/15.0036

Perú, Ministerio de Agricultura y Riego (2017). Series históricas de producción agrícola- Compendio estadístico. Recuperado de http://frenteweb.minagri.gob.pe/ sisca/?mod=consulta_cult

Moya, P.F.; Dawe, D.; Pabale, D.; Tiongco, M.; Chien, N.V.; Devarajan, S.; Djatiharti, A.; Lai, N.X.; Niyomvit, L.; Ping, H.X.; Redondo, G. y Wardana, P. (2004). The economics of intensively irrigated rice in Asia. In Dobermann, A., Witt, C., Dawe, D. (eds.). Increasing productivity of intensive rice systems through site-specific nutrient management. Science Publishers, Inc., Philippines, pp. 29-58 
Organización de las Naciones Unidas para la Alimentación y la Cultura (2017). Base de datos de producción, FAOSTAT. Disponible en: http://www.fao.org/faostat/en/\#data/QC

Ottis, B.V. y Talber, R. (2005). Rice yield components as affected by cultivar and seedling rate. Agronomy journal 97:1622-1625

Pearson, J. y Stewart, G.R. (1993). The deposition of atmospheric ammonia and its effects on plants. New Phytol 125:283-305. doi:10.1111/j.1469-8137. 1993.tb03882.x

Pampolino, M.F.; Manguiat, I.J.; Ramanathan, S.; Gines, H.C.; Tan, P.S.; Chi, T.T.N.; Rajendran, R. y Buresh, R.J. (2007). Environmental impact and economic benefits of site-specific nutrient management (SSNM) in irrigated rice systems. Agric. Syst. 93:1-24

Quintero, C.; Zamero, M.; Van Dendonckt, G.; Boschetti, G.; Befani, M.; Temporetti, C.; Arévalo, E.; Spinelli, N. y Panozzo, J. (2014). Momentos oportunos para fertilización con nitrógeno en arroz en Entre Ríos, Argentina. IAH-13-marzo. Facultad de Ciencias Agropecuarias-UNER, Fundación PROARROZ. CC 24 Paraná ER (3.100)

Ramírez, G.E. y Sánchez, P.A. (1971). Factores que afectan la eficiencia de utilización del nitrógeno en arroz bajo riego intermitente. Programa Nacional de Arroz. Perú. Inf. Tec., pp. 50 -51

Roberts, T.L. (2008). Improving nutrient use efficiency. Turk. J. Agric. For. 32: 177-182

Shrawat, A.K.; Carroll, R.T.; DePauw, M.; Taylor, G.J. y Good, A.G. (2008). Genetic engineering of improved nitrogen use efficiency in rice by the tissue-specific expression of alanine aminotransferase. Plant Biotechnol J: 6:722-32; PMID:18510577; http://dx.doi. org/10.1111/j.1467-7652.2008.00351.x

Schnnier H. F., Dingkuhn M., De Datta S. K., Mengel K., Faronilo J. E., (1990). Nitrogen fertilization of direct seeded flooded vs transplanted rice: I. Nitrogen uptake, photosynthesis, growth and yield. Crop Sci. 30:1276-1284.

Singh, H.; Verma, A.; Wahid A., M. y Shukla, A. (2014). Physiological response of rice (Oryza sativa L.) genotypes to elevated nitrogen applied under field conditions. Plant Signaling \& Behavior 9, e29015, Landes Bioscience 
Snyder, C. (2009). Eficiencia del uso de nitrógeno: Desafíos mundiales, tendencias futuras. Informaciones agronómicas, 75

Somansundaram, E.; Velayutham, A.; Poonguzhalan, R. y Sathiyavelu, A. (2002). Effect of nitrogen levels on growth and yield rice (SSRC 91216, TRY 2) under sodic soil conditions. Mad Agric. J; 506-8

Thompson, H. (2012). Food science deserves a place at the table - US agricultural research chief aims to raise the profile of farming and nutrition science. Nature, doi:10.1038/ nature.2012.10963

Yoshida, S. (1981). Fundamentals of Rice Crop Science. International Research Institute, Philippines, pp. 269

Youngdahl, L. J.; Lupin, M.S. y Craswell, E.T. (1986). New developments in nitrogen fertilizers for rice. Fertilizer Research 9: 149-160 